\title{
On a mathematical model in estimating the reliability of technological equipment by non- destructive testing
}

\author{
Vyacheslav Berkovich ${ }^{1, *}$,Victor Poltinnikov ${ }^{2}$ \\ ${ }^{1}$ Don Cossacks' State Institute of Food Industry and Business (brunch) «MGUTU» named after \\ K.G.Razumovsky (First Cossacks' University), 55, Semashko, Rostov-on-Don, 344007, Russia \\ ${ }^{2}$ Don State Technical University, 1 Gagarin sq., Rostov-on-Don, 344010, Russia
}

\begin{abstract}
The mathematical model to describe the acoustic emission (AE) in a massive elastic body by radiating piecewise linear defect at the stage of its extension is considered. Problems of reconstructing the wave field in the body and analyzing characteristics of the defect by AE testing are discussed. The problem in question is reduced to the investigation of a boundary integral equations system. The model presented allows to raise the reliability of non-destructive testing results when estimating the exhaustible resource of equipment under long exploitation as one of the directions to solve the problem of energy saving and efficiency.
\end{abstract}

\section{Introduction}

Problems of estimating and predicting the exhaustible resource of the objects of technological equipment being in exploitation have been always of great interest for authorities. Problems mentioned above are of especial urgency when the replacement of the obsolete equipment by more modern one meets the serious spending that sometimes may be non-evidenced to producing purposes.

Contemporary ways for analyzing the industry objects of responsible purposes under exploitation consist in using non-destructive testing techniques [1] in various worldwide spheres of diagnostics of objects such as industrial and civil buildings, industrial piping systems, concrete bridges, nuclear power facilities [2-7] at al. Among these techniques the acoustic emission (AE) method takes the especial place when estimating the predestructive stages of exploited equipment material since AE method permits to detect growing defects being just the most dangerous[8,9].

In the paper suggested the dynamic problem of steady vibrations arising in the massive elastic body is considered in a pre-destructive stage of its material under exploitation. The physical process of the acoustic radiation is studied in the stage of growing the inner defect appeared earlier under loadings and formatting the tree graph-shaped defect (Fig.1). The only element of the defect resulted by growing the former one is assumed to be radiated that is well-known from experiments. The problem of the wave field reconstruction in the body is stated. Defects are simulated by linear cuts of finite length.

*Corresponding author: bvn06@yandex.ru 
The mathematical statement of the problem in question leads to the mixed boundary value problem for the Helmholtz equation in the half-space with the radiating cut $J$. The statement is reduced, in turn, to the equivalent system of boundary integral equations (BIA). Solvability problems of equations are examined and the structure of its solution is established.

To estimate radiating defect parameters, to fulfill mathematical modeling the wave field depending on radiating type, form and location of inner defect with its radiation characteristics. The simulation of the AE process described above is assumed to be a number of steps. It may be considered $\mathrm{AE}$ as determined process under antiplane deformation or under plane deformation or under $3 \mathrm{D}$ deformation or considered $\mathrm{AE}$ as stochastic process ets.

At present paper we consider but the initial step of modeling the AE process in a massive body under antiplane deformation in spite of existing the translational symmetry as the accessory product of that type of simulation. However, it should be noted that such a model, first hand, use the simple mathematical technique and, from the other hand, allows to obtain the main qualitative results of investigating the problem in question. This modeling consists in the consideration of elastic antiplane oscillations by the inner tree graph-shaped defect $J$ growing to the free boundary of a massive body.

In the Fig. 1 it is shown the defect $A_{0} A_{1} A_{2} A_{11} A_{12} A_{21} A_{22}$ with its possible localization presented by the active radiating element $A_{0} A_{1}$ and the passive rest $A_{1} A_{2} A_{11} A_{12} A_{21} A_{22}$ of no radiating. The situation described above generates the mathematical model of the $\mathrm{AE}$ process within the chosen pre-failure stage.

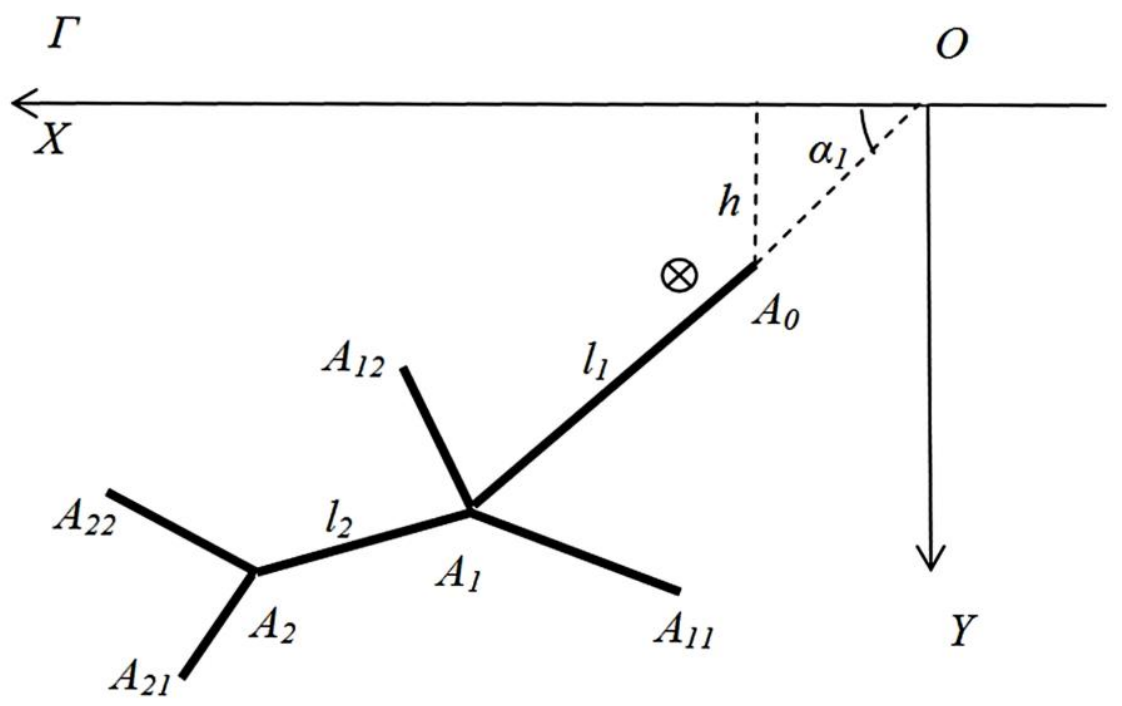

Fig. 1. Radiating defect $J$ in a massive body, $\bigotimes$ - oscillations of the element $A_{0} A_{1}$ are perpendicular to $(x, y)$ plane

The massive body is simulated by the elastic half-space $\Omega$ with unloaded boundary $\Gamma$. Radiating defect is presented by the broken cut $A_{0} A_{1} A_{2}$ (Fig.2) consisting of active 
radiating element $J_{1}=A_{0} A_{1}$ and the passive one $J_{2}=A_{1} A_{2}$ of finite lengths $l_{1}, l_{2}$ with inclination angles $\alpha_{1}, \alpha_{2}$ to the boundary $\Gamma$ respectively.

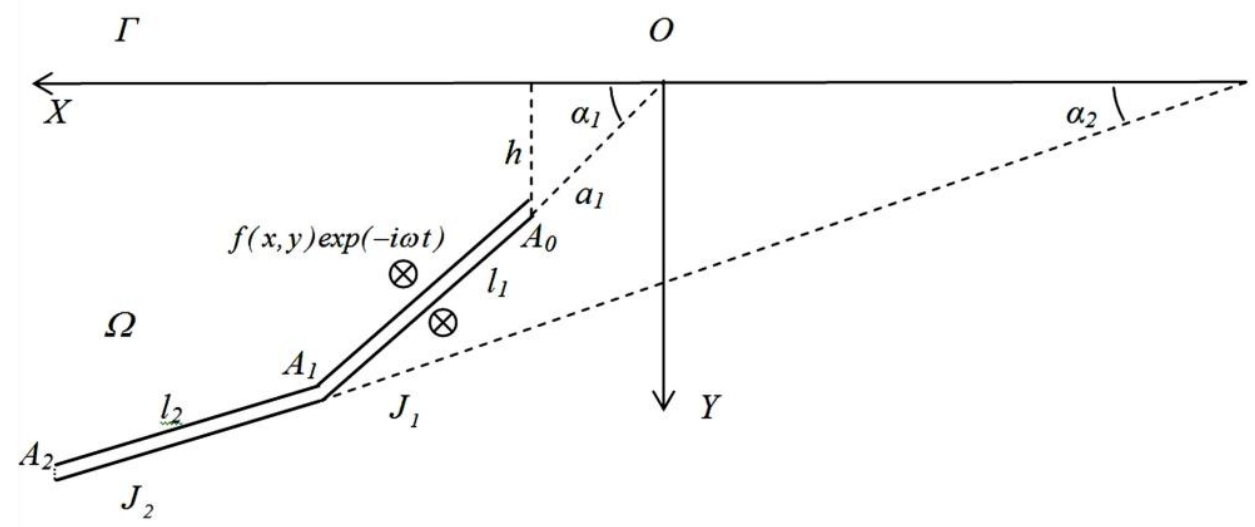

Fig. 2. Radiating broken cut $J=J_{1} \cup J_{2}$

The point $A_{0}$ being the nearest to the free boundary $\Gamma$ is remote from it on the distance $h$. Shear displacements harmonic oscillation generators are located but on cut boundaries $J_{1}^{ \pm}$, ones are coherent, have the equal intensity, are given and distributed as $f_{l}(x, y) \exp (-i \omega t),(x, y) \in J_{l}^{ \pm}, \omega$ is the circular frequency. Displacements of the cut boundaries $J_{2}^{ \pm}$are assumed to be equal zero. Amplitudes of outgoing displacement waves in $\Omega$ vanish at infinity. The problem in question is to reconstruct the wave displacements field in the whole $\Omega$ under conditions listed above.

\section{Problem statement}

To solve the problem in question, to consider differential equations of the dynamic elasticity [10] in the half-space $\Omega$ with unloaded boundary $\Gamma$ and cuts $J_{1,2}$ of finite length forming the cut $J$. Representing the displacement oscillations in the form

$$
W(x, y, t)=u(x, y) \exp (-i \omega t)
$$

we reduce the problem above to the mixed boundary value one about the unknown displacement complex amplitude $u(x, y)$ in $\Omega$ as follows :

$$
\begin{aligned}
& \Delta u+k^{2} u=0, \quad k^{2}=\omega^{2} D \mu^{-1}, \\
& \left.\frac{\partial u}{\partial v}\right|_{\Gamma}=0, \quad[u]_{J}=0, \quad \frac{\partial u}{\partial v}-i k u=0\left(\frac{1}{\sqrt{r}}\right), \quad r=\sqrt{x^{2}+y^{2}} \rightarrow \infty \\
& \left.u\right|_{J^{ \pm}}=f(x, y),\left.\quad u\right|_{J^{ \pm}}=0, \quad(x, y) \in J
\end{aligned}
$$

where $D$ is the material density, $\mu$ is the shear modulus, $v$ is the exterior normal to the boundary $\partial \Omega,[u]_{J}$ is the function saltus via the cut $J$. In the formulae (2) conditions as $r \rightarrow \infty$ are Somerfield radiation ones $[11,12]$ of waves propagation to the infinity where its 
amplitudes vanish. The solution of the boundary value problem (2) is considered as an element of Sobolev space $W_{2}^{1}(\Omega)$ [13].

The method of investigating the mixed boundary value problem (2) is based on reducing one to the corresponding boundary integral equation system by constructing Green function $G(x, y \mid \xi, \eta)$ for Helmholtz equation. Green function obeys the condition $\partial u /\left.\partial v\right|_{\Gamma}=O$ and Somerfield radiation condition (2). Hereby, there takes place the representation for the complex amplitude in the form of curvilinear integrals along right boundaries of cuts $J_{1,2}^{+}$:

$$
u(x, y)=\sum_{n=1}^{2} \int_{J_{n}^{+}} G(x, y \mid \xi, \eta) q_{n}(\xi, \eta) d l, \quad(x, y) \in \Omega
$$

In the formula (3) functions $q_{n}(x, y)=\mu^{-1}[\partial u / \partial v]_{J_{n}}$ are dimensionless stress saltus via $J_{n}, n=1,2$ respectively. The unknown displacement field may be reconstructed after finding $q_{1,2}(x, y)$.

Let us place the observation point $(x, y)$ on the cut boundaries $J_{1,2}^{+}$and go over to the local coordinates along the boundaries $J_{1,2}^{+}$. To describe further results let us introduce the vector-function $\mathbf{f}(r)$ as the extension of vector-function $\mathbf{f}_{l}(r)=\left(\begin{array}{ll}f_{l}(r), 0<r<l_{1} \\ 0, & 0<r<l_{2}\end{array}\right)$ on the interval $O<r<l=\max \left\{l_{1}, l_{2}\right\}$. Then we result the system of boundary integral equation (BIA) about unknown $q_{1,2}(\rho)$ as follows:

$$
\begin{gathered}
\mathbf{K q}=\int_{0}^{l} \mathbf{k}(r, \rho) \cdot \mathbf{q}(\rho) d \rho=\mathbf{f}(r), \quad 0<r<l \\
\mathbf{k}(r, \rho)=\left(\begin{array}{ll}
k_{11}(r, \rho) & k_{12}(r, \rho) \\
k_{21}(r, \rho) & k_{22}(r, \rho)
\end{array}\right), \quad k_{m n}(r, \rho)=K_{0}\left(\kappa R_{m n}^{-}\right)+K_{0}\left(\kappa R_{m n}^{+}\right), \kappa=-i k \\
\quad(m, n=1,2) \\
R_{11}^{-}=R_{22}^{-}=|r-\rho|, \quad R_{11}^{+}=\sqrt{\left(r+a_{1}\right)^{2}+(\rho)=\left(\begin{array}{l}
q_{1}(\rho) \\
q_{2}(\rho)
\end{array}\right)} \\
R_{12}^{-}=\sqrt{\left(l_{1}+a_{1}-r\right)^{2}+\left(\rho+a_{1}\right)^{2}+2\left(l_{1}+a_{1}-r\right)\left(\rho+a_{1}\right) \cos \left(\alpha_{2}-\alpha_{1}\right)} \\
R_{12}^{+}=\sqrt{\left(r+R_{0}\right)^{2}+\left(a_{1}+\rho_{0}+\rho\right)^{2}-2\left(r+R_{0}\right)\left(a_{1}+\rho_{0}+\rho\right) \cos \left(\alpha_{1}+\alpha_{2}\right)} \\
R_{21}^{-}=\sqrt{\left(l_{1}+a_{1}-\rho\right)^{2}+\left(r+a_{1}\right)^{2}+2\left(r+a_{1}\right)\left(l_{1}+a_{1}-\rho\right) \cos \left(\alpha_{2}-\alpha_{1}\right)} \\
R_{21}^{+}=\sqrt{\left(r+a_{1}+\rho_{0}\right)^{2}+\left(\rho+R_{0}\right)^{2}-2\left(r+a_{1}+\rho_{0}\right)\left(\rho+R_{0}\right) \cos \left(\alpha_{1}+\alpha_{2}\right)} \\
R_{22}^{+}=\sqrt{\left(r+L_{2}\right)^{2}+\left(\rho+L_{2}\right)^{2}-2\left(r+L_{2}\right)\left(\rho+L_{2}\right) \cos 2 \alpha_{2}}
\end{gathered}
$$




$$
\begin{aligned}
& R_{0}=\frac{l_{1} \sin ^{2} \alpha_{2}}{\sin \left(\alpha_{1}-\alpha_{2}\right) \sin \left(\alpha_{1}+\alpha_{2}\right)}, \quad L_{2}=\frac{l_{1} \sin \alpha_{1} \sin \alpha_{2}}{\sin ^{2}\left(\alpha_{1}-\alpha_{2}\right)} \\
& \rho_{0}=l_{1} \sin \alpha_{1} \sin \alpha_{2} \frac{2 \sin \alpha_{1} \cos \alpha_{2}}{\sin ^{2}\left(\alpha_{1}-\alpha_{2}\right) \sin \left(\alpha_{1}+\alpha_{2}\right)}, a_{1}=\frac{h}{\sin \alpha_{1}}
\end{aligned}
$$

In expressions (4) it is taken into account that $q_{1,2}(\rho)=0$ out of the cuts, $\hat{E}_{p}(z)$ is McDonald function [14], $h$ is the distance between the vertex $A_{0}$ of the cut $J_{1}$ and free boundary $\Gamma$. Quantities $R_{0}, L_{2}, \rho_{0}$ appear when constructing Green function by reflection method known in diffraction theory [12].

\section{Results and discussions}

Using summation formulae for McDonald functions permits to transform BIA system (4) to the form:

$$
\begin{gathered}
\mathbf{K q}(\rho)=\int_{0}^{l} \mathbf{k}(r, \rho) \cdot \mathbf{q}(\rho) d \rho=\mathbf{f}(r), \quad 0<r<l \\
\mathbf{k}(r, \rho)=\mathbf{h}(r, \rho)+\mathbf{s}(r, \rho) \\
\mathbf{h}(r, \rho)=\frac{1}{\pi} \int_{-\infty}^{+\infty} K_{-i u}(\kappa r) K_{-i u}(\kappa \rho) \mathbf{H}(u) d u, \quad \kappa=-i k \\
\mathbf{s}(r, \rho)=\frac{1}{\pi^{2}} \int_{-\infty-\infty}^{+\infty} \int_{-i \eta}^{+\infty} K_{-\infty}(\kappa r) K_{-i \eta^{\prime}}(\kappa \rho) K_{-i\left(\eta-\eta^{\prime}\right.}(\kappa M) \mathbf{S}(\eta) d \eta d \eta^{\prime} \\
\mathbf{H}(u)=\mathbf{H}\left(u \mid \alpha_{1}, \alpha_{2}\right), \mathbf{S}(u)=\mathbf{H}_{1}\left(u \mid l_{1}, l_{2}, \alpha_{1}, \alpha_{2}\right), M=M\left(l_{1}, l_{2}, \alpha_{1}, \alpha_{2}\right)
\end{gathered}
$$

Expressions of components of matrixes $\mathbf{H}, \mathbf{H}_{1}$ are not adduced due to there awkwardness. It points out the left hand side of operator $\mathbf{K}$ in (5) may be represented as the sum $\mathbf{K}=\mathbf{H}+\mathbf{S}$ generated by summands of the kernel $\mathbf{k}(r, \rho)=\mathbf{h}(r, \rho)+\mathbf{s}(r, \rho)$. Hereby, operator $\mathbf{H}$ is uniquely inverted and operator $\mathbf{S}$ is compact as operators acting in Sobolev-Slobodetsky spaces $W_{2}^{ \pm 1 / 2}\left(J_{n}^{+}\right), n=1,2$ of the fractional smoothness.

Solution structure of BIA system (5) may be taken by [15] and has the representation as follows ( $T$ is transpose operator):

$$
\begin{aligned}
\rho \mathbf{q}(\rho) & =\frac{1}{\pi i} \int_{\Gamma_{l}} \mathbf{H}^{-1}(z) \cdot \mathbf{F}_{l}(z) I_{-i z}(\kappa \rho) z d z+\frac{1}{\pi i} \int_{\Gamma_{2}} \mathbf{H}_{-}^{-1}(z) \cdot \mathbf{X}(z) I_{-i z}(\kappa \rho) z d z \\
\mathbf{H}(z) & =\mathbf{H}_{-}(z) \cdot \mathbf{H}_{+}(z), \quad \mathbf{F}_{1}^{T}(z)=\left\{\int_{0}^{l_{l}} f_{l}(r) K_{-i z}(\kappa r) r^{-1} d r, 0\right\} \\
\mathbf{X}(z) & \in S_{\sigma}\left(\Gamma_{2}\right), \sigma>\gamma, 1<\gamma<2, \quad \mathbf{X}^{T}=\left\{X_{1} \cdot K_{-i z}\left(\kappa l_{1}\right), X_{2} \cdot K_{-i z}\left(\kappa l_{2}\right)\right\}
\end{aligned}
$$

Contour $\Gamma_{2}$ is located upper $\Gamma_{1}\left(\Gamma_{1,2} \subset \Pi\right)$ in the complex z-plane, $X_{1,2}(z)$ are unknown functions, $I_{-i z}(k r), K_{-i z}(k r)$ are modified Bessel functions [14], $S_{\sigma}\left(\Gamma_{2}\right)$ is 
the space of vector-functions $\mathbf{X}(z)$, converging to zero together with the pour weight $\mathrm{z}^{\sigma}$ in a regularity strip $\Pi \supset R^{1}$ of integrand matrix-function $\mathbf{H}(z)$. Matrix-functions $\mathbf{H}_{ \pm}(z)$ are resulted by the factorization $\mathbf{H}(z)$ with respect to real axis $R^{l}$.

The proof of the representation (6) is based on results [15]. Subsequent substituting the solution (6) to the BIA system (5) adduces to the auxiliary integral equations system of the second type with compact operator in the space $S_{\sigma}\left(\Gamma_{2}\right), \sigma>3 / 2$ for searching the unknown vector-functions $\mathbf{X}(z)$. It permits to make well-known conclusions about the solvability of the system.

Compactness of the operator of that system allows to represent its operator as the sum of finite-dimensional and infinitesimal ones. This fact permits to apply various numerical methods to solve the equations with compact operators and hence to solve input boundary value problem (2).

Reconstructing the wave field in the whole domain $\Omega$ with the defect $J$ may be fulfilled by the representation (3) and solution (6) of BIA system (5). In part, to reconstruct the wave field in the free boundary $\Gamma$, to tend the observation point $(x, y)$ to $\Gamma$ in the representation (3). Fig.3 illustrates the result of evaluating the $\operatorname{Re} u(x, y),(x, y) \in \Gamma$ in the form of the amplitude-frequency response of free boundary $\Gamma$ of the half-space containing defect $J$ (Material is Steel 20).

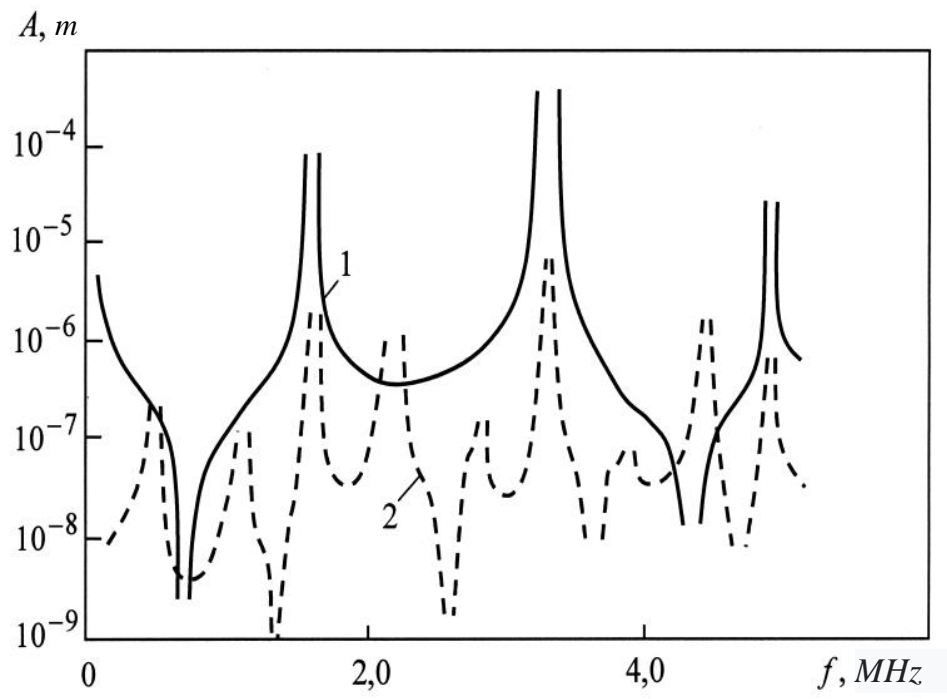

Fig. 3. Frequency response for free-surface displacement amplitude:

(1) $\alpha=45^{\circ}, l_{1,2}=0,5 \cdot 10^{-4} \mathrm{~m}$; (2) $\alpha=45^{\circ} l_{1,2}=1,5 \cdot 10^{-3} \mathrm{~m}$

The infinite amplitude values according to resonance frequencies are caused by the ideal elastic material in applied mathematical model. Furthermore, using the least square method allows to solve the inverse problems of reconstructing the unknown radiation amplitudes of the defect $J$ by ones measured on the free boundary $\Gamma$ on the base of AE diagnostics [16-19]. 


\section{Conclusions}

1. Mathematical model offered to study AE process by piecewise linear defect allows to consider process of growing and accumulating the defects.

2. Investigating the model of $\mathrm{AE}$ process in details permits to connect theoretical results with experimental data on processing and analyzing AE signals detected on the free boundary of a body.

3. The way offered may be applied to consider inverse problems of reconstructing unknown physical parameters of the process of growing inner defects of a massive body on the base of analyzing the AE signals.

4. The development of the method presented may become the base on considering consequent stages of the mathematical simulation $\mathrm{AE}$ under plane and $3 \mathrm{D}$ deformation of the material both in determined process variant and the stochastic one.

5. The method offered allows to consider the problem of energy saving and efficiency by timely and reliable estimating the exhaustible resource of the technological equipment to prolong its exploitation.

\section{References}

1. I.N. Ermolov, N.P. Aleshin, A.I. Potapov, Acoustic Testing Methods. Ser.: NonDestructive Testing. Higher School Press, (1991)

2. K. K. Kong, S. Noroozi at al, Nondestr. Testing and Evaluation, 29(2), (2014)

3. Yap Huey Tyng, Ong Zhi Chao at al, J. of Mech. Sci. and Tech., 31(8), (2017)

4. I. Zubaidah, I. Zainah at al, J. of Bridge Eng., 17(6), (2012)

5. Ong Zhi Chao, Hoe Cheng Eng at al., Engineering Failure Analysis, 82, (2016)

6. S. K. Loh, F. Waleed at al, Science China Technological Sciences, 54(5), (2011)

7. Ayo-Imoru Ronke, A.C. Cilliers, Annals of Nuclear Energy 112, (2018)

8. V.I. Ivanov, and V.A.Barat, Acoustic-Emission Diagnostics. Moscow: Spektr, 2017.

9. S.I. Builo, Physical, Mechanical and Statistical Aspects of Acoustic Emission Diagnostics. In: Physics and Mechanics of New Materials and Their Applications. New York: Nova Science Publishers. 2013 (Chapter 15).

10. V.G.Rekach, Guide to Solving Problems in the Theory of Elasticity, Moscow: Higher School Press, 1977.

11. V.N.Berkovich, A.V. Babkin, Int. Res. J, 4 (2017)

12. M.Sh.Israilov, Dynamic Elasticity and Wave Diffraction, (Moskow State University, Russia, 1977)

13. O.V.Besov, V.P.Il'in, S.M.Nikolskii, Integral Representations of Functions and Embedding Theorems, Moscow: Science Press, 1975

14. N.N. Lebedev, Special Functions and Their Applications, Moscow-Leningrad: Science Press, 1968.

15. V.N.Berkovich, Rep. Acad. Sci. USSA, 267,17 (1982).

16. S.I.Builo, Rus.J. Non-Destr. Testing, 11 (2012)

17. V.G.Romanov, Inverse Problem of Mathematical Phisycs, Moskow: Science Press, 1984

18. A.O.Vatulyan, Inverse Problems in Mechanics of Deformable Bodes, Moskow: Phys.Math. Lit.Press, 2007. 
19. A.O. Vatulyan, A.N.Solovyev, Direct and Inverse Problems for Homogeneous and Inhomogeneous Elastic and Electro-elastic Bodies (Southern Federal University, Rostov-on-Don, Russia, 2008). 